\title{
Suizid: höchste Zeit zu handeln oder - bleibt es ein Dauerbrenner?
}

There is no health without mental health (WHO 2001)

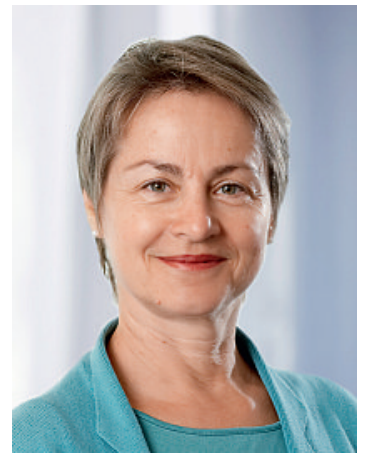

Christine Romann
Bereits 1991 begann das nationale FMH-Fortbildungsprogramm mit den Worten «Die Schweiz ist ein Land mit hoher Suizidrate. Mehr als die Hälfte der Menschen, die Suizid begehen, sucht in den der Suizidhandlung vorausgehenden Tagen und Wochen einen Arzt auf. (...) Wir möchten mit Ihnen zusammen erreichen, dass die Zahl der Suizidhandlungen in unserem Land abnimmt, und sich Ärztinnen und Ärzte frei und kompetent fühlen, Patienten während der Sprechstunde auf emotionale Probleme und Suizidgedanken anzusprechen (...)».

Heute, fast 20 Jahre später, besteht nach wie vor Handlungsbedarf. Die Zahlen sind erschreckend: Ca. 1000 Männer und 400 Frauen suizidieren sich jedes Jahr. Für Männer zwischen 15 und 44 Jahren ist der Suizid die häufigste Todesart überhaupt. Und mit einer Suizidrate von 19,1 (pro 100000 Einwohner) ist die Schweiz weit vorne auf einer unrühmlichen Liste. Wir müssen also Wege finden, dies zu ändern.

Eine suizidale Handlung hat immer eine Geschichte, und in diese sind Ärztinnen und Ärzte häufig involviert. Jede Krise, welche die Bewältigungsstrategien eines Menschen überfordert, kann in eine suizidale Entwicklung münden. Bis zum Suizid vergehen Wochen bis Monate, in denen diese Menschen Hilfe suchen - auch bei ihrer Haus-
Natürlich sind es zuvorderst die Psychiater; Suizidalität ist eine schwierige und konstante Begleiterin durch ein psychiatrisch-psychotherapeutisches Arbeitsleben. Betroffen ist aber auch die Gynäkologin, deren Patientin mit einer postpartalen Depression kämpft; der Orthopäde, der den Patienten mit chronischen Schmerzen im Wartezimmer hat; die Lehrerin, der ein Jugendlicher mit Schulproblemen auffällt; der Seelsorger, der den frisch verwitweten Rentner betreut sie alle haben eine Chance, die Betroffenen aus ihrer suizidalen Entwicklung rauszuholen, aber wie? Es geht um Leben oder Tod, um Verzweiflung und Wut, um Resignation und Hass. Das Thema Suizidalität geht unter die Haut und löst auch grosse eigene Ängste und Unsicherheiten aus.

\section{Der Arzt muss vor allem die Depressionen erkennen}

Wie kann man Suizidalität erkennen? Wen behandelt man wie? Was darf, soll oder muss man tun, wo sind juristische Stolpersteine, wie kann die Zusammenarbeit zwischen psychiatrischen Spezialisten und Grundversorgern weiter verbessert werden, wie können die hohen Eintrittsschwellen für adäquate psychiatrische Dienstleistungen sowohl für die Betroffenen als auch für deren Angehörige gesenkt werden? Der nationale Dachverband IPSILON für Suizidprävention bearbeitet diese Fragen am Nationalen Suizid-Kongress, wel-

\section{Jede Krise, welche die Bewältigungsstrategien eines Menschen überfordert, kann in eine suizidale Entwicklung münden}

ärztin. 90 Prozent dieser Patienten haben eine psychiatrische Diagnose, dennoch sind vorab die Hausärztinnen und Hausärzte gefordert, denn sie sind es, die von vielen im Vorfeld eines Suizides aufgesucht werden. Der Hausarzt muss vor allem die Depressionen erkennen - auch dann, wenn sich der Patient selber über die psychische Natur seiner Beschwerden nicht im Klaren ist. Auch Personen aus anderen Fachrichtungen sind mit Depression und Suizidalität konfrontiert. cher am 18./19. März 2010 in Zürich stattfindet. Die FMH begrüsst und unterstützt den Dachverband IPSILON und seine Aktivitäten. Denn - es ist höchste Zeit zu handeln!

Informationen unter: www.ipsilon.ch

Dr. med. Christine Romann, Mitglied des Zentralvorstands der FMH, Verantwortliche Ressort Gesundheitsförderung und Prävention 